\title{
»Alles ist möglich dem, der glaubt!« versus »Ich glaube, hilf meinem Unglauben!»
}

»Ich glaube, hilf meinem Unglauben!« ist der verzweifelte Ausruf eines Vaters, dessen Kind krank ist, und der Jesus um Hilfe bittet. Sein Ruf steht in einer Wundererzählung im Markusevangelium (Mk 9,24). Diese gibt Einblick in das Glaubensverständnis jenes Evangeliums und in seine theologische Reflexion über Glaube und Unglaube (1.). Wie verhalten sich die widersprüchlichen Größen Glaube und Unglaube zueinander? Wie kann Glaube ungläubig sein und Unglaube zum Glauben werden? Um diesen Fragen nachzugehen, soll die Wundererzählung in Mk 9,14-28 mit dem Doppellogion vom sprichwörtlich Berge versetzenden Glauben und dem Bittgebet in Mk 11,22-25 verglichen werden. Anhand dieser Texte soll veranschaulicht werden, dass Mk das Gebet als Hinwendung zu Gott versteht, in dem der Unglaube zum Glauben vordringt und darauf vertraut, dass Gott Bitten erhört (2.). Abschliessend wird ein Resümee gezogen (3.).

\section{»Ich glaube, hilf meinem Unglauben«. Ein »Gebetsruf« im Kontext von Mk 9,14-28}

Im paradoxen Gebetsruf aus Mk 9,24 spricht sich die Gleichzeitigkeit von Glaube und Unglaube aus. Er steht im Kontext einer Wundererzählung, in der es um die Heilung eines epileptischen Kindes geht $(9,14-28)$. In ihr verbinden sich gattungstypische Elemente der Therapien und Exorzismen. ${ }^{1}$ Spannungen und Doppelungen innerhalb der Erzählung veranlassten zu literarkritischen Trennungen und der Annahme, es handele sich um ursprünglich zwei eigenständige Überlieferungen. ${ }^{2} \mathrm{Pesch}^{3}$ hat dagegen überzeugend die Einheitlichkeit der Erzählung aufgezeigt. Redaktionelle Eingriffe seitens des Evangelisten verdanken sich der Platzierung der Wundererzählung im Mikrokontext sowie im Gesamtaufbau des

1 R. Pesch, Das Markusevangelium (HThKNT II/2), Freiburg i. Br. 2001, 95; s. ausführlich P. Dschulnigg, Das Markusevangelium (ThKNT), Stuttgart 2007, 252.

2 Vgl. die ausführliche Wiedergabe der Diskussion bei J. Gnilka, Das Evangelium nach Markus (EKK II/2), Neukirchen-Vluyn 1979, 44 f.

3 Pesch, Das Markusevangelium, 85. 
Evangeliums, und Spannungen sowie Doppelungen erklären sich aus dem intendierten Erzählverlauf. ${ }^{4}$

InV. 14 setzt mit einem Ortswechsel ein neuer Erzählabschnitt ein. Jesus und drei seiner Jünger werden Zeugen eines Konflikts zwischen den übrigen Jüngern und Schriftgelehrten. Eine Menschenmenge hatte sich um die Diskutanden gebildet. Diese strömt nun Jesus entgegen und begrüsst ihn. Es wird erzählt, dass sie sich aufgrund der Gegenwart Jesu entsetzten (V. 15). Damit entspricht das Verhalten der Menschenmenge der Reaktion der Menschen auf Jesu erstes lehrendes und heilendes Auftreten $(1,27)$. Der Erzähler gibt damit den Hinweis, dass das im folgenden Geschilderte im Lichte des bislang berichteten Wirkens Jesu und seiner Reich Gottes Verkündigung verstanden werden will. ${ }^{5}$

Die VV. 16-18 bilden den Auftakt eines Dialogs, der in den Redeteilen Jesu zunächst die Menge einbezieht und sich später zu einem Zwiegespräch zwischen Jesus und dem Vater des kranken Kindes entwickelt (VV. 21-24). Dieser nennt auf die Frage Jesu hin den Grund der Auseinandersetzung. Er hatte die Jünger gebeten, den sprachlosen Geist ( $\pi v \varepsilon \tilde{v} \mu \alpha$ ő $\lambda \alpha \lambda \mathrm{\alpha} v$ V. 17) hinauszuwerfen, der sein Kind quält, aber den Jüngern fehlte die Kraft dazu. ${ }^{6}$ Jesus begründet das Jüngerversagen mit dem Unglauben (V. 19), der jedoch nicht

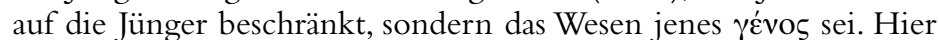
scheint eine grundsätzliche Beurteilung durch: Das Problem des Unglaubens wird als allgemein menschliches Phänomen benannt, das in der Ablehnung Jesu und seinerVerkündigung als Angebrochensein des Reiches Gottes besteht.

\footnotetext{
4 So ist nun das Subjekt in V. 9,14 aus der vorangehenden Erzählung von der Verklärung Jesu (9,2-13) in Anwesenheit dreier seiner Jünger übernommen, während es in der vormarkinischen Überlieferung auf den Wundertäter allein bezogen war. Die Jünger werden in V. 14 als in sich undifferenzierte Gruppe indirekt doppelt genannt. Sie treten damit in den Fokus des Interesses.Vgl. auch Pesch, Das Markusevangelium, $86 f$.

5 F. Hahn, Das Verständnis des Glaubens im Markusevangelium, in: Ders./H. Klein (Hg.), Glaube im Neuen Testament (BThSt 7), Neukirchen-Vluyn 1982, $43-67$ (54f.).; O. Hofius, Die Allmacht des Sohnes Gottes und das Gebet des Glaubens, ZThK 101 (2004), 117-137, hier 117.

6 Als sperrig erweist sich die zweifache Schilderung vom Bringen des Kindes zu Jesus in den VV. 17 und 20, die bei Mt und Lk fehlt. Wird sie nicht mit der Annahme begründet, es handele sich um eine ursprünglich eigenständig überlieferte Bitte um Heilung einer anderen Symptomatik (vgl. die erneute Krankheitsdarstellung in V. 20), kann sie aus der Erzählabsicht in V. 17 als Fehlverhalten erklärt werden, das in V. 20 eine Korrektur erhält: der Kranke soll zu Jesus statt zu den Jüngern gebracht werden. Vgl. O. Hofius, Die Allmacht des Sohnes Gottes und das Gebet des Glaubens, 122.
} 
Nachdem Jesus gefordert hat, das Kind zu ihm zu bringen (V. 19), erscheint der eingeschaltete Dialog zwischen ihm und dem Vater (VV. 21-24) als retardierendes Moment: die Heilung bleibt aus, stattdessen kämpft der Junge gegen die Symptome. Diese entsprechen dem Krankheitsbild der Epilepsie (V.20), die vor dem Hintergrund einer mythologisch-metaphorischen Vorstellungswelt als Besessenheit aufgefasst wurde. In Mk 9,14-28 wird sie auf einen sprachlosen und unreinen Geist zurückgeführt. ${ }^{7}$ Dieser reagiert auf die Begegnung mit Jesus aktiv $(9,20)$ und entspricht damit dem bei Mk beschriebenen Verhalten von Dämonen, die die Identität Jesu erkennen. ${ }^{8}$

Die zweifache Schilderung der Krankheit (VV. 18. 21f.) pointiert die Hoffnungslosigkeit. Anstelle des Kranken rückt der ungläubig glaubende Vater ins Zentrum der Erzählung. Die Heilung selbst wird erst nach der erzählerischen Klimax beschrieben (VV. 25-27), d.h. erst nach der Aussage Jesu »Alles ist möglich dem, der glaubt!» und dem Aufschrei des Vaters »Ich glaube, hilf meinem Unglauben! « (V. 23f.). Die Hervorhebung des Unglaubens der Jünger und der Schriftgelehrten sowie die zentrale Stellung des Dialogs Jesu mit dem zweifelnden Vater lässt die Wunderhandlung in den Hintergrund treten. Im Fokus steht eine theologische Reflexion über Glaube und Unglaube, ${ }^{9}$ deren Rahmen die Wundererzählung bildet.

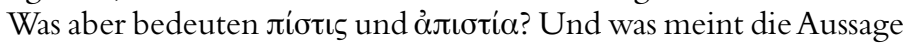
"Alles ist möglich dem, der glaubt!«(V. 23)? Um diese Fragen zu klären, soll zunächst das Gespräch zwischen Jesus und dem Vater (VV. 22-24) betrachtet werden.

\footnotetext{
7 J. N. Neumann, Besessenheit, in: K. Scherberich (Hg.), Neues Testament und Antike Kultur, Bd. 2: Familie - Gesellschaft - Wirtschaft, Neukirchen-Vluyn 2005, 71-74, hier 71; M. Ebner, Jesus von Nazaret in seiner Zeit (SBS 196), Stuttgart 2003, 71. Der antike Volksglaube stellte sich Besessenheit als Ergriffensein der Person von personalen Größen dar. Sie werden mit Dämonen und Totengeistern identifiziert. Man nahm an, diese gelangten über Körperöffnungen in den Menschen, was auf die mythologisch gedeutete Erfahrung zurückgehen dürfte, dass Parasiten in den Körper eindringen und Menschen krank machen.Vgl. weiter R. von Bendemann, Christus als Arzt. Krankheitskonzepte in den Therapieerzählungen des Markusevangeliums (Teil I), in: BZ (54/1) 2010, 36-53; (Teil II), in: BZ (54/2) 2010, 162-178.

8 Mk 1,25.34; 3,12.

9 Das belegen das gehäufte Vorkommen an Derivaten der $\pi \iota \sigma \tau-$ bzw. $\alpha \pi \iota \sigma \tau-$ Stämme

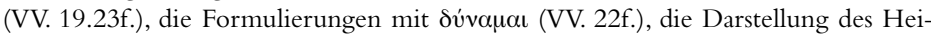

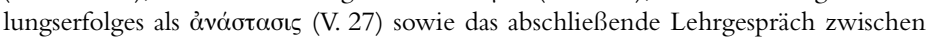
Jesus und den Jüngern, das das Gebet als Seinsmodus glaubender Existenz benennt (VV. 28f.).
} 


\section{1»Ich glaube, hilf meinem Unglauben!« in der Konfrontation mit: »Alles ist möglich dem, der glaubt!«}

Angesichts der Not des Kindes fragt Jesus den Vater,seit wann der Junge unter der Krankheit leide (V. 21). Die Antwort des Vaters "seit seiner Kindheit« kann ebenso wie die drastische Krankheitsschilderung auf die Zweifel an einer möglichen Heilung hinweisen. ${ }^{10}$ Das scheint auch die konditional formulierte an Jesus gerichtete Bitte des Vaters auszudrücken: "Wenn du aber etwas vermagst, hilf uns«(V. 22). Noch einmal klingt die enttäuschte Hoffnung an, dass die Jünger und Schriftgelehrten nicht dazu in der Lage waren, dem Kind zu helfen. ${ }^{11}$ Zudem gibt die Verzweiflung des Vaters Einblick in die schwierige soziale Situation in einer Gesellschaft, in der Epilepsie als Besessenheit gedeutet und auf Dämonen zurückgeführt wurde. Die Krankheitssymptome konnten Außenstehende derart verunsichern, dass die Erkrankten von der Gesellschaft ausgeschlossen wurden. Das Neue Testament erzählt häufig von Besessenen, deren Leiden auch als Spiegelbild einer Gesellschaft gedeutet werden können, die unter Fremdherrschaft und Einfluss verschiedener Kulturen stand. ${ }^{12}$

Während sich der Vater von den Jüngern noch eine Austreibung des Geistes erhofft hatte (V. 18), erbittet er von Jesus nur noch Hilfe und Mitleid. Jesus kommentiert diese Bitte als Ausdruck mangelnden Vertrauens: Er nimmt die Worte des Vaters auf - "wenn du kannst" - und konfrontiert sie gleich darauf mit der Aussage "Alles ist möglich, dem der glaubt!« (V. 23). In diesem Moment brechen sich die Emotionen des Vaters Bahn. Er schreit auf: »Ich glaube, hilf meinem Unglauben!« Damit ist die Klimax des Gesprächs erreicht: DerVater

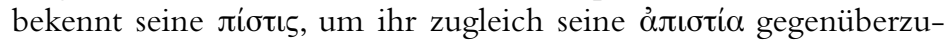
stellen (V. 24). ${ }^{13}$ Statt die heilende Macht Jesu für möglich zu halten, überbietet die Negation jedes Glaubens den Zweifel. Dennoch klingt in dem Gebetsruf des Vaters auch der Wunsch an, »ich will vertrauen, verhelfe mir dazu, meinen Zweifel zu überwinden «. ${ }^{14}$ Die

\footnotetext{
${ }^{10}$ R. Pesch, Das Markusevangelium, 91.

${ }^{11}$ G. Theissen, Urchristliche Wundergeschichten (StNT 8), ${ }^{7} 1998$, 139f. Einen anderen Aspekt hebt Hofius, Die Allmacht des Sohnes Gottes und das Gebet des Glaubens, 123-126, hervor. Er versteht $\tau$ als adverbialen Akkusativ, so dass die Frage des Vaters "im Sinn eines emphatischen swenn irgend möglich der nachdrücklichen Unterstreichung einer Bitte" (124) diene. Sie sei daher nicht Ausdruck des Zweifels, sondern "Ruf aus der Tiefe äußerster Not« (126).

12 Theissen, Urchristliche Wundergeschichten, 248.Vgl. den Besessenen von Gerasa (Lk 8, 26-39), dessen Dämon Legion genannt wird.

${ }^{13}$ Vgl. die übrigen ỏंı

${ }^{14}$ H. F. Bayer, Das Evangelium des Markus, HTA, Witten 2008, 337.
} 
folgende Heilung des Kindes ereignet sich nun unabhängig vom Glauben des Vaters.

»Alles ist möglich dem, der glaubt!« Ist diese Aussage auf Jesus zu beziehen? Oder fordert dieser den Vater auf zu glauben? Auf wen

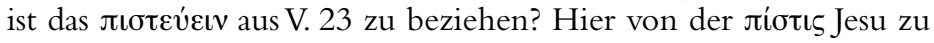
sprechen, wird zuweilen mit dem Hinweis abgelehnt, dass an keiner anderen Stelle im Markusevangelium der Glaube Jesu ausgesagt sei. ${ }^{15}$ Der Aufbau der Erzählung allerdings legt nahe, dass es sich in V. 23 um eine Selbstaussage Jesu handelt. Er nimmt die an ihn gerichtete zweifelnde Anfrage des Vaters wiederholend auf und bezieht das Vermögen $\mathrm{zu}$ heilen auf sich. "Jesu Wirken tritt also in ein ganz spezielles Licht: Er ist nicht nur der, mit dem Gottes eschatologische Herrschaft verwirklicht wird und der Gottes Handeln in Gang setzt, er ist gleichzeitig derjenige, der den Menschen in ihrer Glaubensnot gegenüber Gott helfen und beistehen soll. ${ }^{16}$ Jesus erscheint hier als der Stellvertreter Gottes auf Erden, der menschgewordene Gott. Mit seiner Zuwendung zu den Menschen, hier als Heilung erzählt, verwirklicht er Gottes Willen. Der Glaube Jesu ist das gehorsame Sich-Unterstellen ${ }^{17}$ unter den Heilswillen Gottes. Die Evangelisten verwenden für diese Deutung ihrer Sicht der Geschichte Jesu zuweilen Zitate aus dem Alten Testament. So kommentiert Mk die Heilung eines Taubstummen mit einem Zitat aus dem Propheten Jesaja $(35,5)$ : „Gut gemacht hat er alles, die Tauben macht er hören und die Stummen sprechen. "Aufgrund dieser Deutung des Handelns Jesu als Bevollmächtigter Gottes, erkennt der zweifelnde Vater in Jesus "Quelle und Grund" des Glaubens. Jesu Glaube manifestiert sich darin, dass er sich »der Wirklichkeit Gottes preisgibt und [...] darauf [...] seine Existenz gegründet sein läßt [...] und die ganze Existenz zum Einsatz ${ }^{18}$ bringt.

${ }^{15}$ G. Barth, Glaube und Zweifel in den synoptischen Evangelien, ZThK 72 (1975), 267-292, hier 279f. G. Ebeling, Jesus und Glaube, ZThK 55 (1958), 64-110, hier 98, hingegen geht von einer Identifikation Jesu mit dem von ihm verkündigten Glauben aus.

${ }^{16}$ Hahn, Das Verständnis des Glaubens im Markusevangelium, 59.Vgl. Ebeling, Jesus und Glaube, 101.

${ }^{17}$ Von Gehorsam spricht auch Ebeling, ebd..

${ }^{18}$ Ebeling, ebd. Etwas anders argumentiert Hofius, Die Allmacht des Sohnes Gottes und das Gebet des Glaubens, 127-129, dessen Aussageintention aber letztlich ver-

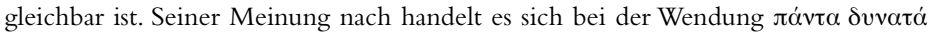
um eine "geprägte Formulierung" (128) für die Allmacht Gottes, zudem bestimmt er

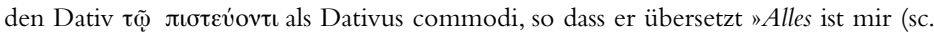
Jesus) möglich - dir zugute, wenn du glaubst. « 
Hat der Vater Jesus missverstanden, wenn er ruft »Ich glaube, hilf meinem Unglauben!« und Jesu Selbstaussage als Zusage und Aufforderung an jeden Glaubenden versteht? Die paradoxe Reaktion des Vaters stellt keineswegs ein Missverstehen der Antwort Jesu dar. ${ }^{19}$ Im Ineinander von Glaube und Unglaube erfährt derVater, dass Jesu Glaube und sein Vertrauen in Relation zueinander stehen. Jedoch nicht in dem Sinne, dass der Glaube des Vaters die Bedingung

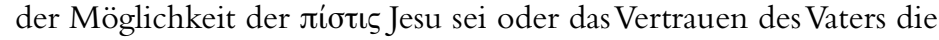
Ursache für die erzielte Wirkung der Heilung darstellte. Das existenziell Ermutigende und Befreiende der Wundererzählung besteht in der Aussage, "dass der Glaube keine menschliche Möglichkeit ist, sondern dem Menschen durch den rettenden Christus entlockt wird" und er "damit ihrer Suche nach Hilfe die Eindeutigkeit des Glaubens zuerkennt. ${ }^{20}$ Im Ringen nach Glauben erkennt der Vater, dass Christus sich insofern vom Vertrauen des Menschen abhängig macht, als dass der Glaube eine Beziehung zwischen Retter und Gerettetem begründet. Diese Relation betrifft die gesamte Existenz des Menschen, zu der die Erfahrung von Glaube und Unglaube gleichermaßen gehören. Bornkamm beschreibt Glaube daher treffend als "Widerspiel alles Zweifelns", als »ein Geschehen zwischen Macht und Ohnmacht «. ${ }^{21}$

Anders als die Jünger und Schriftgelehrten gelangt der Vater gerade im Unglauben zum Glauben. Er vertraut darauf, dass er sich in seinem Unglauben an Christus wenden kann, weil dieser die "Wirklichkeit Gottes in der Welt verkörpert. ॥ $^{22}$ Mit dem paradoxen Bekenntnis ungläubigen Glaubens richtet der Vater seine Existenz auf denjenigen aus, der es vermag, Glauben hervorzurufen und mit Unglauben konfrontiert zu werden..$^{23}$ "Ich glaube, hilf meinem Unglauben! « kann daher als Gebetsruf bezeichnet werden.

In diesem Sinne kommentiert $\mathrm{Mk}$ in 9,28f. auch die erfolgte Heilung. Er lässt die Erzählung mit einer Belehrung der Jünger durch Jesus schliessen, in der das Gebet als Ermöglichungsgrund der Heilung genannt wird. Das Gebet ist verstanden als charakteristi-

\footnotetext{
19 So Barth, Glaube und Zweifel in den synoptischen Evangelien, 280.

${ }^{20} \mathrm{H}$. Weder, Glaube-Hoffnung-Vertrauen. Thesen zu Beobachtungen aus neutestamentlich-hermeneutischer Perspektive (erscheint in: I.U. Dalferth/S. Peng-Keller (Hrsg.), Theologische Vertrauensfragen. Die ökumenische Diskussion um den Glauben als fiducia, Freiburg i. Br. 2012 (Quaestiones disputatae); Manuskript, 4.

${ }^{21}$ G. Bornkamm, Jesus von Nazareth (UTB 19), Stuttgart ${ }^{15} 1995,121$.

22 Weder, Glaube-Hoffnung-Vertrauen, Manuskript, 1.

${ }^{23}$ Analog hierzu kann Paulus vom Glauben an den Gott sprechen, der den Gottlosen rechtfertigt. Der Mensch erkennt sich erst in der Begegnung mit Jesu Jíotıs als ungläubig (vgl. auch Gal 2,16 bzgl. der Erkenntnis des Sünderseins aller Menschen).
} 
scher "Ausdruck des Glaubens, der sich vertrauend auf das Wirken Gottes verläßt $\aleph^{24}$ - und, wie der Evangelist Mk zeigt - ebenso der Unglaube Ausdruck finden kann.

Mit der Erwähnung des Gebets schafft Mk eine Überleitung zu einer weiteren Reflexion über Glaube und Unglaube bzw. Zweifel, in der dem Gebet eine zentrale Rolle zukommt. Das Doppellogion vom Berge versetzenden Glauben und dem Bittgebet in Mk 11,24f. hat viele Parallelen mit der Wundererzählung in Mk 9,14-28: Glaubensvollmacht versus Unglaube; Zweifel und Gebet werden thematisiert.

Aufgrund der begrifflichen, inhaltlichen sowie strukturellen Übereinstimmungen mit der besprochenen Wundererzählung, soll im folgenden das Doppellogion vom »Berge versetzenden« Glauben und dem Bittgebet betrachtet werden.

\section{Das Doppellogion vom Berge versetzenden ${ }^{25}$ Glauben und dem Bittgebet}

Das Logion vom Berge versetzenden Glauben ist fester Bestandteil der synoptischen Überlieferung. Darüber hinaus findet sich eine motivliche Allusion bei Paulus (1 Kor 13,2). Der ursprüngliche Wortlaut des Logions kann nicht rekonstruiert werden. Im Markusevangelium hat es folgende Gestalt:

22 Und Jesus antwortete ihnen und sagte: Habt Glauben an Gott! 23 Amen, ich sage euch: Wer zu diesem Berg spräche: Werde weggetragen und ins Meer geworfen, und nicht zweifelte in seinem Herzen, sondern glaubt, dass geschieht, was er sagt, dem wird es zuteil werden. (Mk 11,22f.)

Während Mt und Lk konditional formulieren, ${ }^{26}$ stellt $\mathrm{Mk}$ den Worten Jesu über den Berge versetzenden Glauben den Imperativ "Habt Glauben an Gott« (V. 22) voran. Das zeigt, dass "Glaube sein eigentliches Wesen in der Beziehung auf ein Gegenüber hat. Die Substanz des Glaubens ist dessen Relation. ${ }^{27}$ Der Vergleich der

${ }^{24}$ Hahn, Das Verständnis des Glaubens im Markusevangelium, 50 Anm. 36.

${ }^{25}$ Der griechische Text spricht von einem Berg (Sg.), der sich emporhebt, d.h. entwurzelt wird. Aufgrund des metaphorischen Gebrauchs bei Mk übernehme ich die im Deutschen sprichwörtliche Rede vom Berge versetzenden Glauben.

${ }^{26}$ Vgl. den detaillierten synoptischen Vergleich bei F. Hahn, Jesu Wort vom bergeversetzenden Glauben, ZNW 76 (1985), 149-169, hier 149-157.

${ }^{27}$ Weder, Glaube-Hoffnung-Vertrauen, Manuskript, 1. 


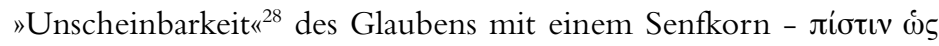

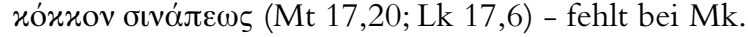

Es fällt auf, dass Mk und Mt dem Glauben den Zweifel gegenüberstellen. Der matthäische und markinische Text wird damit transparent für eine Reflexion über Glaube und Zweifel: Beide adressieren ihren Text an die Jünger, d.h. aus nachösterlicher Perspektive an die christliche Gemeinde. ${ }^{29}$ Beide Evangelisten lokalisieren damit das Verhältnis Glaube und Zweifel nicht etwa in einem missionarischen Kontext oder als Kriterium zur Differenzierung zwischen Glaubenden und Nicht-Glaubenden. Schauplatz des Gegeneinanders von Glaube und Zweifel ist das Individuum, wie beim Vater in der Wundererzählung, ${ }^{30}$ als auch die Gemeinschaft der ersten Christen. Darüber hinaus verbinden Mt und Mk das Logion vom Berge versetzenden Glauben mit dem Logion vom Bittgebet. ${ }^{31}$ Der Mk-Text lautet:

24 Darum sage ich euch, alles worum ihr betet und bittet, glaubt, dass ihr es empfangen habt, und es wird euch zuteil werden. 25 Und wenn ihr steht und betet, vergebt, wenn ihr etwas gegen jemanden habt, damit auch euer Vater in den Himmeln euch eure Verfehlungen vergibt. (Mk 11,24f.)

Ein Blick auf den Aufbau der Evangelien zeigt, dass die Synoptiker die Logien ihrer jeweiligen Aussageintention entsprechend in den Gesamtaufbau ihres Evangeliums integriert haben. Änderungen und Einfügungen erhellen das jeweilige Glaubensverständnis. ${ }^{32}$

Bei Mk stehen sowohl 9,23f. als auch das Logion vom Berge versetzenden Glauben im Kontext eines Wunders, ${ }^{33}$ das sich auf das voll-

\footnotetext{
${ }^{28}$ Ebeling, Jesus und Glaube, 92.

${ }^{29}$ Vgl. zur matthäischen Konzeption des Kleinglaubens, der gerade erst in der Nachfolge zur Diskussion steht: Barth, Glaube und Zweifel in den synoptischen Evangelien, 282-290.

${ }^{30} \mathrm{Vgl}$. den verzweifelten Ausruf des Vaters Mk 9, 24 als individuelles Bekenntnis, in: der ein Ich spricht.

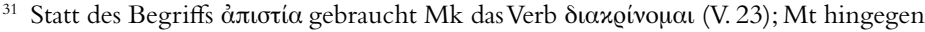
spricht einmal von ỏ $\lambda \iota \gamma o \pi \iota \sigma \tau i \alpha$, in Verbindung mit dem Bittgebet, dann ebenfalls vom Zweifel (Mt 21,21).

${ }^{32}$ So überliefern $\mathrm{Mk}$ und $\mathrm{Mt}$ das Logion im Zusammenhang mit der Verfluchung des Feigenbaumes (Mk 11,22-14; Mt 21,21f.), Mt darüber hinaus im Kontext der Heilung des epileptischen Kindes (Mt 17,20), während es bei Lk als eigenständiges Logion erscheint, das statt eines Berges von einem Maulbeerfeigenbaum spricht, der entwurzelt wird (Lk 17,6).

${ }_{33}$ Aufgrund der Annahme, dass $\mathrm{Mk}$ die ursprünglich eigenständigen Logien vom "Berge versetzenden" Glauben und dem Bittgebet zu einer Einheit verbunden hat, konzentriere ich mich auf die Exegese dieser Logien und vermeide eine kontextuelle
} 
mächtige Wort Jesu hin ereignet (11,12-14). Die Glaubensvollmacht bezieht sich wiederum auf Jesus selbst, wird aber nun auf die Glaubenden übertragen. Damit werden die Glaubenden in das Gottesverhältnis Jesu aufgenommen und partizipieren, wie dieser, an der Allmacht Gottes. Das Bild vom Berge versetzenden Glauben kann also als Synonym zur paulinischen und johanneischen Konzeption der Gotteskindschaft gelten. ${ }^{34}$ Zudem repräsentiert es eine Art "Gruppenmessianismus", der sich in der Fortführung der vollmächtigen Verkündigung durch die Jünger realisieren soll. ${ }^{35}$ Und dennoch wirkt das Logion befremdlich. Das Bergmotiv klingt gewaltig, und selbst die metaphorische Deutung bereitet Schwierigkeiten: Dem Glauben wird die Ermöglichung des Unmöglichen zugesagt. Die Spannung hebt sich auch nicht auf, wenn das Logion als "proverbialtype saying " verstanden wird, analog zu Jesu Rede vom Kamel, das durch ein Nadelöhr gelangt. ${ }^{36}$ Das Wort vom Berge versetzenden Glauben ist unglaublich, lässt zweifeln. Ähnlich unglaublich erscheint das Logion vom Bittgebet. Es verallgemeinert, was das Logion vom Berge versetzenden Glauben mit Hilfe eines Bildes aus der Natur

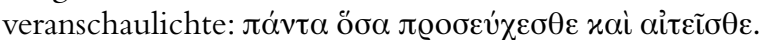

Ein Blick in den griechischen Wortlaut bietet Hilfe: Der Tempus-

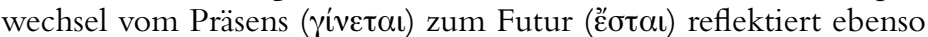
wie der Aorist $\dot{\varepsilon} \lambda \alpha \dot{\beta} \varepsilon \tau \varepsilon$, dass das im Glauben Erbetene nicht unmittelbar eintritt bzw. sich entgegen allen Augenscheins bereits realisiert hat, ohne dass die Betenden es wahrgenommen hätten. ${ }^{37}$ Dadurch wird ein Glaubensverständnis verhindert, das die Empirie als Allmachtsphantasie entlarven würde. Zentral ist die Ermutigung zum Glauben, wie der Imperativ (V. 24) und die Kontrastformulierung "und nicht zweifelte in seinem Herzen, sondern glaubte» (V. 23) betonen. Der Zweifel widerspricht dem Glauben, indem er den

Deutung im Zusammenhang mit dem Fluchwunder gegen den Feigenbaum und der Tempelreinigung. Solch eine Interpretation steht in der Gefahr, "antijudaistische Tendenzen in das NT « einzuschreiben, indem der verfluchte Feigenbaum mit Israel identifiziert wird und als Metapher für die Tempelreinigung fungiert. Das Motiv des "Berg versetzenden" Glaubens würde dann vor dem Hintergrund alttestamentlicher und jüdischer Tradition mit dem richtenden Handeln Gottes verbunden. Hiervor warnt zu Recht Pesch, Das Markusevangelium, 135, mit dem Hinweis, dass der "Feigenbaum sonst nie selbständiges Symbol für Israel« sei.

34 So auch Gnilka, Das Evangelium nach Markus, 135.

${ }^{35}$ Begriff von G. Theissen, Gruppenmessianismus. Überlegungen zum Ursprung der Kirche im Jüngerkreis Jesu, JBTh 7 (1992), 101-123.

${ }^{36}$ R.T. France, The Gospel of Mark (The New International Greek Testament Commentary), Grand Rapids 2002, 448.

${ }^{37}$ Der Aorist betont hier den ingressiven Aspekt. Gegen Gnilka, Das Evangelium nach Markus, 135, der eine futurische Bedeutung annimmt. 
Menschen wie seinVertrauen auf Gott spaltet. ${ }^{38}$ Die Formulierungen zeugen von der theologischen Reflexion des Mk ebenso wie von der Erfahrung der ersten Christen. Darin erhält der Text seine aktuelle Bedeutung: Die Ermutigung zum Glauben hat paränetische Funktion, sie weiß von der Existenz des Zweifels und der Verzweiflung, derWeltwirklichkeit, die der Botschaft vom Reich Gottes widerspricht.

Wie schon in der Wundererzählung wird das Gebet als "Lösungsstrategie« genannt. Die Aufforderung zum vertrauensvollen Bittgebet

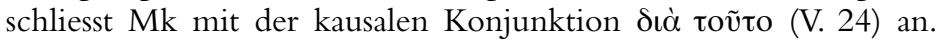
Somit erklärt Mk das Gebet zu jener Ausdrucksform, in der der »ungläubige Glaube« darauf vertraut, dass sein Zweifel von der Macht Gottes überwunden wird, indem sich der Beter ebendieser Macht und Wirklichkeit Gottes anvertraut.

Allerdings ist das Gebet kein geschlossener Raum zwischen Gott und Mensch. Es reisst den Menschen nicht aus seinen sozialen Bezügen und entbindet ihn nicht von seiner Verpflichtung gegenüber dem Mitmenschen. Wie Christus in den Wundererzählungen als jemand geschildert wird, der die konkrete Not der Menschen wahrnimmt und der aus dem Gebet Kraft zur Heilung empfängt, ${ }^{39}$ wird auch hier das Gebet an ein ethisches Kriterium gebunden: die Bereitschaft, einander zu vergeben. Die zwischenmenschliche Vergebungsbereitschaft ist die Bedingung dafür, dass Gott die Verfehlungen der Menschen vergibt $(11,25)$. Betender Glaube beschreibt ein »uneingeschränktes Sich-Verlassen und Sich-Hingeben, das dem Menschen Anteil an Gottes eigenem Wirken gewährt « ${ }^{40}$, so dass "die Macht des Glaubens" die "Macht Gottes" ist. ${ }^{41}$ Solchem Gebetsglauben als "Gott-in-Aktion-treten-Lassen «" ${ }^{42}$ ist die Erhörung immer schon vorweggenommen, da seine "Gewißheit $[\ldots]$ die Gewißheit und Macht Gottes selbst ist", wie »die Wahrheit und Wirklichkeit« der Worte Jesu »die Wahrheit und Wirklichkeit Gottes ist. « ${ }^{43}$

\footnotetext{
38 Pesch, Das Markusevangelium, 205.

${ }^{39} \mathrm{Mk}$ 7,31-37; Joh 11,1-44.

${ }^{40}$ Hahn, Das Verständnis des Glaubens im Markusevangelium, 53.

${ }^{41}$ Ebeling, Jesus und Glaube, 96.

${ }^{42}$ Ebd.

${ }^{43}$ Ebd., 97.101.
} 


\section{3. «Alles ist möglich dem, der glaubt!« versus »Ich glaube, hilf meinem Unglauben!«?}

Die besprochenen Texte aus dem Markusevangelium provozieren: Un-glaublich klingen die Worte »Alles ist möglich dem, der glaubt! « Viel vertrauter tönt der betende Vater: "Ich glaube, hilf meinem Unglauben!« Ein genauerer Blick auf die Texte will versuchen, die Provokation zu fassen.

Die narrative Analyse der Wundererzählung in Mk 9,14-28 hat gezeigt, dass hier von der Glaubensvollmacht Jesu die Rede ist. Mk erzählt mit einem christologischen Impetus: seine Adressaten sollen in Jesus den erkennen, in dem Gottes Wesen sichtbar wird (vgl. Mk 1,1). Er ist es, der Glauben hervorrufen kann und sich trotz des Unglaubens nicht abwendet. In seiner Zuwendung zu den Menschen realisiert sich Gottes Heilshandeln. Die erfahrene Zuwendung deutet $\mathrm{Mk}$ als Befreiung zum Leben und Auferstehung $(9,27)$.

Der Gebetsruf des Vaters »Ich glaube, hilf meinem Unglauben!« veranschaulicht, dass im Gebet auch »ungläubiger Glaube «Ausdruck finden kann und Glaube und Unglaube einander nicht ausschlieBen.

"Alles ist möglich dem, der glaubt!« überträgt das Doppellogion vom Berge versetzenden Glauben und dem Bittgebet in Mk 11, 22-25 von Jesus auf die Glaubenden. Ihnen wird verheissen, dass sie wie Jesus an der Allmacht Gottes partizipieren, und ihnen alles, worum sie bitten, zuteil geworden sei. Ein Blick auf die Tempora hat gezeigt, dass Glaube und Gebet keine Allmachtsphantasien stillen wollen. Stattdessen kann die Partizipation an der Allmacht Gottes als Aufnahme in das Gottesverhältnis Jesu verstanden werden. Das Logion vom Berge versetzenden Glauben stellt die Analogie der Synoptiker zur paulinischen und johanneischen Konzeption der Gotteskindschaft dar. Glaube ist in der Beziehung zu Gott realisiert.

"Alles, worum ihr betet und bittet, glaubt, dass ihr es empfangen habt, und es wird euch zuteil werden" (Mk 11,24). Diese Zusage Jesu provoziert; sie widerspricht der Weltwirklichkeit, den Erfahrungen der ersten Christen ebenso wie den unsrigen. Das scheint auch dem Evangelisten Mk für seine Zeit bewusst gewesen zu sein. Erneut ist es seine christologische Darstellung, die eine hermeneutische Reflexion dieser provokanten Zusage ermöglicht.

Mk lenkt den Blick seiner Adressaten auf Jesus in Gethsemane. Hier ist es Jesus, der betet. Trotz seines Vertrauens auf die Allmacht 
Gottes $(14,36),{ }^{44}$ hat er Todesangst. Nach dem Markusevangelium stirbt er als Gottverlassener: $\varepsilon \lambda \omega \mathrm{\iota} \varepsilon \lambda \omega \mathrm{\iota} \lambda \varepsilon \mu \alpha \sigma \alpha \beta \alpha \chi \theta \alpha v \mathrm{\iota}(15,34)$ - ein Ausruf des Zweifels und des Unglaubens?! Jesus solidarisiert sich hier mit den Menschen bis in den Tod und die Gottverlassenheit hinein. Mit dieser theologia crucis vertritt das Markusevangelium einen Vertrauensglauben, der der Wirklichkeit von Leid und Tod nicht flieht und sich nicht an Wundern messen lässt. Die Erzählungen des Mk veranschaulichen, dass das Schicksal Jesu wie das des Vaters nicht in Leid und Tod enden.Vielmehr verheisst der Glaube Leben (16,6; 9,27).

Das Markusevangelium ermutigt zum Gebet als Ausdrucksform "ungläubigen" wie auch vertrauenden Glaubens, indem es Jesus als Betenden beschreibt und die Glaubenden als von Jesus zum Gebet Aufgeforderte. Die Forderung, nicht zu zweifeln, hat paränetische Funktion.

Und: Das Gebet ist an ein ethisches Kriterium gebunden: die gegenseitige Vergebungsbereitschaft $(11,25)$. Darin findet sich ein Kern urchristlicher Überzeugung, wie auch die paulinische Parallele vom Motiv des Berge versetzenden Glaubens die Liebe zum Maßstab macht (1 Kor 13,2).

So wollen die behandelten Texte des Markusevangeliums ermutigen zu Glaube und Gebet als Vertrauen auf das "Wirken-Lassen des Rettenden ", ${ }^{45}$ und sie ermöglichen die Identifikation mit der paradoxen Gestalt des Vaters, der unter der Weltwirklichkeit leidet und betet: »Ich glaube, hilf meinem Unglauben!«

— Nadine Kessler ist wissenschaftliche Assistentin am Lehrstuhl für Neutestamentliche Wissenschaft mit Schwerpunkt Antikes Judentum und Hermeneutik an der Universität Zürich.

${ }^{44}$ Anstelle der Allmacht Jesu oder der Glaubenden ist hier wie in Mk 10,27 von der Allmacht Gottes die Rede 14,36.

45 Weder, Glaube-Hoffnung-Vertrauen, 2 